\title{
ESTIMACIÓN DE EROSIÓN POTENCIAL BAJO DOS SISTEMAS DE CORTE DE CAÑA PANELERA UTILIZANDO LA ECUACIÓN UNIVERSAL DE PERDIDA DE SUELOS
}

\section{ESTIMATION OF POTENTIAL EROSION UNDER TWO SUGARCANE CUTTING SYSTEMS USING THE UNIVERSAL SOIL LOSS EQUATION}

\author{
Jose Luis Tauta Muñoz ${ }^{1}$, Jesús Hernán Camacho-Tamayo ${ }^{2}$, Gonzalo Alfredo Rodríguez Borray ${ }^{3}$
}

\begin{abstract}
${ }^{1}$ Ing. Agrícola, M.Sc., Profesional de Apoyo a la investigación. Corporación Colombiana de Investigación Agropecuaria, AGROSAVIA, Centro de investigación Tibaitatá, Km 14 vía Bogotá-Mosquera, Cundinamarca, Colombia, e-mail: jtauta@ agrosavia.co, (Dhttps://orcid.org/0000-0002-2837-4931; ${ }^{2}$ Ing. Agrícola, M.Sc., Ph.D., Profesor asistente, Facultad de Ingeniería, Programa de ingeniería agrícola. Universidad Nacional de Colombia, Carrera 30 No. 45-03, edificio 214, Bogotá, Colombia, e-mail: jhcamachot@unal.edu.co, iDhttps://orcid.org/0000-0002-7066-369X; ${ }^{3}$ Ing. Agrónomo, Economista Agrario, M.Sc., Investigador Máster. Corporación Colombiana de Investigación Agropecuaria, AGROSAVIA, Centro de investigación Tibaitatá, Km 14 vía Bogotá-Mosquera, Cundinamarca, Colombia, e-mail: grodriguez@agrosavia.co, (Dhttps://orcid. org/0000-0001-6261-7418
\end{abstract}

Rev. U.D.C.A Act. \& Div. Cient. 21(2):405-413, Julio-Diciembre, 2018 https://doi.org/10.31910/rudca.v21.n2.2018.1074

Artículo de acceso abierto publicado por Revista U.D.C.A Actualidad \& Divulgación Científica bajo una licencia Creative Commons CC BY-NC 4.0

\section{RESUMEN}

Con el fin de comparar las pérdidas de suelo en dos sistemas de corte tradicionalmente utilizados en el cultivo de caña de azúcar para la producción de panela, se llevó a cabo un estudio, en el que se realizaron mediciones en campo, utilizando un simulador de lluvia y parcelas de escorrentía, en un vertisol del municipio de Quebradanegra, Cundinamarca, en donde se presentan, simultáneamente, los dos tipos de corte: parejo (tipo de corte en el que se cosecha todo a la vez, al tener una madurez homogénea en el cultivo) y entresaque (sistema en el cual no hay madurez homogénea, por lo que se corta solo un porcentaje de las plantas a la vez). Para estimar las pérdidas de suelo potenciales asociadas a ambos sistemas de corte, se utilizó la Ecuación Universal de Pérdida de Suelos (USLE), obteniendo un factor medio C, asociado al tipo de corte, utilizando los datos determinados en campo y la erosividad de las intensidades de precipitación simuladas. Finalmente, se determinó la erosión potencial anual, bajo los dos sistemas de corte, donde se observó que el sistema de corte por parejo presenta un valor 2,2 veces mayor que el sistema de corte por entresaque. Por otra parte, al comparar las pérdidas potenciales de suelo con un lote recién preparado y sembrado, sin cobertura vegetal, se evidencia la capacidad del cultivo de caña panelera para brin- dar protección al suelo, presentando una erosión potencial 9 veces menor, en el caso del corte por parejo, con respecto a una condición de suelo sin cobertura vegetal.

Palabras clave: erosión hídrica, erosividad, erodabilidad, simulador de lluvia, Saccharum officinarum, vertisol (CAB Thesaurus).

\section{ABSTRACT}

In order to compare soil losses in two cutting systems traditionally used in the cultivation of sugar cane for the production of panela, a study was carried out in which measurements were made in the field using a rainfall simulator and runoff plots in a vertisol of the municipality of Quebradanegra, Cundinamarca, where both types of cut are presented simultaneously: the cut-off system, in which everything is harvested at once having a homogeneous maturity in the crop and the thinning cut system, in which have an inhomogeneous maturity so that only a percentage of the plants is cut at the same time. To estimate the potential soil losses associated with both cutting systems, the Universal Soil Loss Equation (USLE) was used to obtain a mean $C$ factor associated with the type of cut, using the data determined in the field and the erosivity of simulated rainfall intensities. Finally, the anual 
potential losses of soil was determined under the two cutting systems, where it was observed that the cut-off system has an annual potential erosion 2.2 times greater than the thinning cuy system. On the other hand, when comparing the potential losses of soil with a newly prepared and planted lot, without plant cover, the capacity of the sugarcane cane crop is demonstrated to offer a protection to the soil, presenting a potential erosion 9 times smaller, in the case of the cut-off system, with respect to a soil condition without plant cover.

Keywords: water erosion, erosivity, erodibility, rainfall simulator, Saccharum officinarum, vertisol

\section{INTRODUCCIÓN}

La erosión del suelo y su redistribución impulsada por el agua y el viento son procesos naturales, que pueden ser acelerados por actividades antrópicas, por cambios en el uso de la tierra, mala gestión agrícola, deforestación y sobrepastoreo (Dercon et al. 2012). El proceso de erosión puede ser visto como el balance entre la erosividad, que incluye, las fuerzas que causan movimiento y la energía suficiente de producir erosión y la erodabilidad, la cual, es la resistencia del suelo a ser movido (Shakesby, 2011). Factores biofísicos, como el clima, la topografía, la cobertura de la tierra y las características específicas del suelo tienen efectos importantes sobre los procesos de erosión y formación del suelo (Gobin et al. 2004). El principal agente de transporte de partículas livianas de suelo es el agua de escorrentía, que se presenta cuando la velocidad de la aplicación del agua excede la velocidad de infiltración del suelo (Shakesby, 2011).

La erosión, a nivel local, a través de un periodo de tiempo, se puede estimar por medio de la Ecuación Universal de Pérdida de Suelos (Universal Soil Loss Equation o USLE), que expresa la erosión en términos de factores asociados a la energía cinética de la lluvia, a las propiedades hidrofísicas del suelo, a la cobertura, al manejo y a la topografía (Williams, 1975). La existencia de un modelo para la predicción, la evaluación y la medición de la erosión hídrica superficial permite entender el comportamiento de dicho fenómeno, así como establecer recomendaciones de manejo y de control, para reducir su efecto negativo en el desarrollo de cultivos.

Las condiciones biofísicas de distintas regiones, con diversas prácticas de manejo de cultivos, hace necesario ajustar los valores establecidos para condiciones específicas. Tal es el caso del cultivo de caña de azúcar para la producción de panela, que contribuye con cerca del 3,5\% del PIB agrícola nacional y vincula, aproximadamente, a 370.000 personas, convirtiéndose en uno de los cultivos agroindustriales más importantes, en términos sociales y económicos para el país (García et al. 2007) que, hasta el momento, no reportaba factores asociados al tipo de corte para la determinación de erosión potencial en el modelo USLE.

Con el fin de evaluar las pérdidas potenciales de suelos en cultivos de caña panelera, se realizaron mediciones en los dos sistemas de corte tradicionalmente utilizados en el país: el corte por entresaque y el corte por parejo, prácticas que obedecen a factores sociales y económicos. El corte por entresaque, tradicionalmente utilizado por pequeños productores, consiste en recolectar las cañas maduras, dejando en el campo las inmaduras para su posterior cosecha; por su parte, el corte por parejo es aplicado en cultivos tecnificados, donde, debido al crecimiento uniforme de los tallos, alcanzan, al mismo tiempo, su estado óptimo de madurez (García et al. 2007). El presente trabajo de investigación tuvo como objetivos: 1) definir, con base en la ecuación universal de pérdida de suelos (USLE), un factor $C$ asociado a los dos sistemas de corte del cultivo de caña panelera, a partir de mediciones directas de erosión hídrica superficial en campo y, 2) determinar la erosión potencial bajo los dos sistemas de corte, estableciendo qué tipo de sistema de corte ofrece la mejor protección, ante eventos de escorrentía superficial.

\section{MATERIALES Y MÉTODOS}

Localización. Las mediciones de pérdida de suelo, se llevaron a cabo en una finca ubicada en las coordenadas geográficas 56'50,53”, de latitud Norte, $74^{\circ} 18^{\prime} 9,28^{\prime \prime}$, de longitud Oeste altitud, de 932m.s.n.m., en el municipio de Quebradanegra, Cundinamarca, donde se registra una temperatura promedio anual de $21^{\circ} \mathrm{C}$ y una precipitación anual de $1.628 \mathrm{~mm}$. En esta finca, se manejan, tradicionalmente, algunos lotes en corte por entresaque y algunos por parejo, lo que permitió reducir la variación en tipo de suelo, condiciones climáticas y demás covariables, que pudieran afectar los resultados obtenidos de suelo removido, por erosión hídrica superficial.

Selección de lotes de medición. La selección de sitios de muestreo, se definió a partir de la determinación de factores asociados al sistema de corte y la pendiente del terreno. El factor inicial que se tuvo en cuenta fue el tipo de corte (entresaque o parejo), escogiendo lotes con un historial de producción previo, asociado a alguno de los tipos de corte; en el caso de entresaque, se realizaron las mediciones en cultivos con cerca de 20 años de manejo, bajo este tipo de sistema; por su parte, los lotes de corte por parejo, tenían cultivos con 7 a 10 años, con este sistema de corte. Además, se estableció una condición de suelo sin ningún tipo de cobertura vegetal, correspondiente a un lote en preparación, para una siembra nueva (plantilla). Con la definición del sistema de corte, se estableció la inclinación del terreno, donde se identificó, en campo, dos rangos de pendiente: el primero, identificado como P1, correspondiente a terrenos con inclinación 
de 15 a $25^{\circ}$ y, el segundo, denominado P2, referente a lotes con inclinación de 35 a $50^{\circ}$. De esta forma, se definieron dos lotes de distinta pendiente para cada uno de los sistemas de corte y para una condición de suelo sin cobertura vegetal.

Para cada uno de los lotes, se determinaron propiedades físicas y químicas asociadas a la Ecuación Universal de Pérdida de Suelos, como el contenido de limo y de arcilla (por el método de Bouyucos), arena muy fina (por medio de tamizado en húmedo), materia orgánica (por el método de Walkey y Black), la determinación del tipo de estructura (evaluación visual) y del tipo de permeabilidad (a partir de la infiltración básica medida en campo). Se identificó el perfil característico de los lotes de mediciones, estableciendo que el suelo correspondía a un vertisol, Typic Haplusterts isohipertérmico muy fino, determinado por la alta presencia de arcillas expandibles, la presencia de grietas, superficies de deslizamiento, estructuras en forma de cuña, con limitantes de uso, debido a fuertes pendientes, pedregosidad esporádica y buen drenaje natural.

Mediciones de suelo removido en campo. Las mediciones de suelo removido, se llevaron a cabo luego del establecimiento de lotes, utilizando un simulador de lluvia en campo portátil, tipo aspersor, diseñado específicamente para el desarrollo del presente estudio, con tres niveles de intensidad de precipitación, correspondientes a 30,81, 44,76 y $78,76 \mathrm{~mm} \mathrm{~h}^{-1}$, asociados a períodos de retorno de 0,2, 2,4 y 105,8 años, respectivamente, determinados por medio de la curva intensidad frecuencia duración, obtenida a partir de datos pluviométricos de la zona, en la que se realizaron los ensayos. El uso de un simulador de lluvia permitió realizar varias mediciones en el día, sin la necesidad de tener que esperar una precipitación natural.

En cada lote establecido, se definieron cuatro parcelas en acero inoxidable, con dimensiones de $4 \mathrm{~m} \times 2 \mathrm{~m}$, distribuidas de forma aleatoria sobre el terreno, cuya instalación, se realizó de forma tal que se evitara, al máximo, alterar el estado del suelo. En cada una de las parcelas, se tomaron mediciones bajo los tres niveles de intensidad de precipitación, aplicadas de forma seguida, con un intervalo de tiempo de 40 a 60 minutos entre aplicación; además, se hicieron mediciones en el momento justo después del corte y repeticiones a los tres y a los seis meses, con excepción de los lotes correspondientes a plantilla, en los cuales, se realizó una única medición en el tiempo.

La salida de la parcela tuvo una forma de embudo, en la que se adaptó un agujero al nivel del suelo, con el fin de permitir la salida del agua de escorrentía y el suelo arrastrado por la misma. El tiempo de simulación de la precipitación que se llevó a cabo correspondió a 15 min, luego, del cual, se obtenía una mezcla de suelo y agua. En total, se llevaron a cabo
168 mediciones y, de cada una, se obtuvo una muestra de suelo removido por escorrentía superficial, que se extrapoló a $\mathrm{kg} \mathrm{ha}^{-1}$, con el fin de expresar la erosión, en términos de peso de suelo por unidad de área.

Ecuación Universal de Pérdida de Suelos (USLE). El modelo empírico USLE de estimación de erosión hídrica promedio anual fue presentado, en 1960, por Wischmeier y Smith, sobre una base de datos de 10.000 combinaciones de parcelas de escurrimiento-año, bajo lluvia natural (García et al. 2017); además de ser el más difundido universalmente (Gvozdenovich et al. 2017), permite la aplicación de adaptaciones metodológicas para el cálculo de sus factores, aun cuando no se cuenta con todos los datos requeridos (Moreno et al. 2017). La descripción de componentes de la Ecuación Universal de Pérdida de Suelos, se muestra a continuación (Wischmeier \& Smith, 1978):

$$
T=R K C L S P
$$

Ecuación 1

Dónde, $T$ es la pérdida media de suelo, generalmente, determinada de forma anual (expresado en unidades escogidas para $K$ y período abarcado en $\left.R, \mathrm{t} \mathrm{ha}^{-1} \mathrm{año}^{-1}\right) . R$ es el factor de erosividad de la lluvia en $M J \mathrm{~cm} \mathrm{ha}^{-1} \mathrm{~h}^{-1}$, que se determinó de forma directa, para el cálculo del factor $C \mathrm{y}$, de forma indirecta, para la determinación de la erosión potencial anual. $K$ es el factor de erodabilidad del suelo, expresado en $\mathrm{t}$ ha $\mathrm{h}$ $\mathrm{MJ}^{-1} \mathrm{ha}^{-1} \mathrm{~cm}^{-1}$. LS es un factor asociado con la longitud y la inclinación de la pendiente. $C$ es un factor adimensional que se relaciona con la cubierta vegetal y el manejo del cultivo (adimensional). $P$ es un factor adimensional asociado a la técnica de conservación aplicada, que se asume igual a 1 , ya que, en los cultivos de caña, sobre los que se realizó la medición, no se aplica ninguna técnica específica de conservación. El factor $K$ de erodabilidad, se determina por medio de la siguiente expresión (Ecuación 2):

$$
K=1,313 * \frac{\left\{\left[\left(2,1 * 10^{-4}\right) *(12-M O) * M^{1,14}\right]+[3,25 *(S-2)]+[2,5 *(P-3)]\right\}}{100}
$$

\section{Ecuación 2}

Dónde, $M O$ corresponde al contenido de materia orgánica del suelo; $S$ es el código de estructura del suelo, que se determina por observación visual y tacto; $P$ es el código de permeabilidad, que se halló por medio de la infiltración básica determinada en campo, por el método de los anillos concéntricos y $M$ es un factor dado por el producto entre la suma de los contenidos de limo y arena muy fina y la suma de los contenidos de arena (sin discriminación de fracciones) y de limo (Wischmeier \& Smith, 1978).

Código del tipo de estructura del suelo $S$. Las categorías y los códigos empleados son cuatro, a saber: granular muy fina - código 1, granular fina - código 2 , granular media a 
gruesa - código 3, bloques, laminar y masiva - código 4; debido a que la estructura del suelo en los lotes era en bloques, se seleccionó el código 4.

Código del tipo de permeabilidad del suelo P. Para la permeabilidad del suelo, se asignan seis valores o códigos diferentes a saber: 1 - Rápida $\left(>12,7 \mathrm{~cm} \mathrm{~h}^{-1}\right) ; 2$ - Moderada a rápida $\left(6,3\right.$ a $\left.12,7 \mathrm{~cm} \mathrm{~h}^{-1}\right) ; 3$ - Moderada (2 a 6,3cm $\left.\mathrm{h}^{-1}\right) ; 4$ - Lenta a moderada $\left(0,5\right.$ a $\left.2 \mathrm{~cm} \mathrm{~h}^{-1}\right) ; 5$ - Lenta $(0,13$ a $\left.0,5 \mathrm{~cm} \mathrm{~h}^{-1}\right)$ y $6-$ Muy lenta $\left(<0,13 \mathrm{~cm} \mathrm{~h}^{-1}\right)$. Para el caso de las mediciones realizadas en campo, se tuvo en cuenta mediciones de infiltración básica, condición, en la cual, el suelo está saturado, por lo tanto, se asocia este valor con la conductividad hidráulica saturada, que es una medida de la permeabilidad y se efectúa la clasificación en las distintas categorías presentadas. El valor de infiltración básica, así como su respectiva clasificación en las categorías mencionadas, se muestran en la tabla 1.

Tabla 1. Factores K de erodabilidad del suelo para lotes de medición.

\begin{tabular}{|c|c|c|c|c|c|c|}
\hline \multirow{3}{*}{ Parámetro } & \multicolumn{2}{|c|}{ Entresaque } & \multicolumn{2}{|c|}{ Parejo } & \multicolumn{2}{|c|}{ Plantilla } \\
\hline & \multicolumn{2}{|c|}{ Media } & \multicolumn{2}{|c|}{ Media } & \multicolumn{2}{|c|}{ Media } \\
\hline & $\mathrm{S}_{\mathrm{d}^{*}}$ & $\mathrm{CV}^{* * *}$ & $\mathrm{~S}_{\mathrm{d}^{*}}$ & $\mathrm{CV}^{* *}$ & $\mathrm{~S}_{\mathrm{d}^{*}}$ & $\mathrm{CV}^{* *}$ \\
\hline $\begin{array}{l}\text { Código de tipo de } \\
\text { estructura }\end{array}$ & \multicolumn{2}{|c|}{4} & \multicolumn{2}{|c|}{4} & \multicolumn{2}{|c|}{4} \\
\hline \multirow{2}{*}{$\begin{array}{l}\text { Infiltración básica } \\
\qquad\left(\mathrm{cm} \mathrm{h}^{-1}\right)\end{array}$} & \multicolumn{2}{|c|}{25,21} & \multicolumn{2}{|c|}{19,71} & \multicolumn{2}{|c|}{3,23} \\
\hline & 25,60 & 1,02 & 7,51 & 0,38 & 4,28 & 1,32 \\
\hline $\begin{array}{c}\text { Código de tipo } \\
\text { de permeabilidad }\end{array}$ & & & & & & \\
\hline \multirow{2}{*}{$\begin{array}{c}\text { Contenido de arcilla } \\
\qquad(\%)\end{array}$} & \multicolumn{2}{|c|}{44,03} & \multicolumn{2}{|c|}{49,68} & \multicolumn{2}{|c|}{27,17} \\
\hline & 8,97 & 0,20 & 4,81 & 0,10 & 2,73 & 0,10 \\
\hline \multirow{2}{*}{$\begin{array}{c}\text { Contenido de limo } \\
(\%)\end{array}$} & \multicolumn{2}{|c|}{27,33} & \multicolumn{2}{|c|}{19,36} & \multicolumn{2}{|c|}{45,06} \\
\hline & 9,21 & 0,34 & 1,13 & 0,06 & 1,01 & 0,02 \\
\hline \multirow{2}{*}{$\begin{array}{l}\text { Contenido de arena } \\
\text { muy fina } \\
(\%)\end{array}$} & \multicolumn{2}{|c|}{6,57} & \multicolumn{2}{|c|}{4,85} & \multicolumn{2}{|c|}{11,20} \\
\hline & 5,29 & 0,81 & 1,61 & 0,33 & 2,50 & 0,22 \\
\hline \multirow{2}{*}{$\begin{array}{c}\text { Contenido de Arena } \\
\text { fina } \\
(\%)\end{array}$} & \multicolumn{2}{|c|}{4,64} & \multicolumn{2}{|c|}{6,13} & \multicolumn{2}{|c|}{5,00} \\
\hline & 0,67 & 0,14 & 0,68 & 0,11 & 1,20 & 0,24 \\
\hline \multirow{2}{*}{$\begin{array}{c}\text { Contenido de Arena } \\
\text { media (\%) }\end{array}$} & \multicolumn{2}{|c|}{3,88} & & & & \\
\hline & 1,22 & 0,32 & 1,05 & 0,19 & 1,11 & 0,36 \\
\hline Contenido de Arena & & & & & & \\
\hline$(\%)$ & 1,76 & 0,43 & 1,39 & 0,20 & 3,62 & 0,75 \\
\hline Contenido de Arena & & & & & & \\
\hline muy gruesa (\%) & 3,78 & 0,59 & 2,92 & 0,39 & 1,08 & 0,32 \\
\hline & & & & & & \\
\hline & 1190,13 & 0,59 & 162,79 & 0,13 & 119,95 & 0,03 \\
\hline Contenido de Materia & & & & & & \\
\hline Orgánica (\%) & 1,66 & 0,25 & 0,98 & 0,14 & 0,77 & 0,14 \\
\hline Factor de erodabilidad & & & & & & \\
\hline$\left(\mathrm{T}\right.$ ha h ha $\left.{ }^{-1} \mathrm{MJ}^{-1} \mathrm{~cm}^{-1}\right)$ & 0,05 & 0,35 & 0,01 & 0,18 & 0,07 & 0,20 \\
\hline
\end{tabular}

*S (Desviación estándar)

**CV (Coeficiente de variación) 
Los factores $L S$ asociados con características de la pendiente del terreno, se determinaron de manera conjunta por medio de la Ecuación 3, que corresponde a la ecuación descrita por Arnoulds (1977):

$$
L S=\left(\frac{\gamma}{22,1}\right)^{0,6} *\left(\frac{S}{9}\right)^{1,4}
$$

Ecuación 3

Dónde, $\Upsilon$ representa la longitud de la ladera en m y $S$ es el porcentaje de inclinación. Las divisiones por 22,1 y 9 representan las respectivas razones de ladera consideradas sobre la longitud y el grado de pendiente de la parcela estándar USLE. Los factores $L S$, se obtuvieron teniendo en cuenta las condiciones de grado de inclinación de la pendiente de cada una de las parcelas de medición, así como la longitud que, en todos los casos, correspondió a 4,7m. Al reemplazar los valores de inclinación y de longitud medidos en campo en cada una de las parcelas de medición en la Ecuación 3, se obtuvieron los valores $L S$, que correspondieron a una media de 1,1 , para lotes con pendientes de 15 a $25 \%$ y de 3,5, para lotes con pendientes de 35 a 50\%. Panagos et al. (2015) determinaron que en zonas planas el factor $L S$ es de 0,2 a 0,5 y que, según la pendiente, este valor puede alcanzar valores mayores a 10.

Aplicación de la Ecuación para determinar el factor C. Para despejar el factor $C$ en cada una de las mediciones, con el fin de realizar estimaciones de este índice para los sistemas de corte y la plantilla, en los distintos tiempos asociados al corte, se tiene en cuenta un factor $R$, correspondiente a la erosividad de las intensidades de precipitación simuladas en campo, aplicando el método directo tradicional, que inicia con la aplicación de la Ecuación 4:

$$
e=0,119+0,0873 * \log I_{m}
$$

Ecuación 4

Donde, $I_{m}$ es la intensidad de la lluvia medida, para un intervalo de tiempo $m$ y e es la energía cinética de cada milímetro de agua caída en él. Luego, se determina la energía total del intervalo, como se presenta en la Ecuación 5:

$$
E_{i}=e_{i} * p p_{i}
$$

Ecuación 5

Donde, $E_{i}$ es la energía total del intervalo $i ; e_{i}$ corresponde a la energía cinética por milímetro, calculada en la Ecuación 4 y $p p_{i}$ es el total de milímetros de lluvia caídos en el intervalo considerado. Finalmente, se determinó la intensidad máxima en 30 minutos, obteniendo, de esta forma, el factor $R$, como se expresa en la Ecuación 6:

$$
R_{i}=E_{i} *
$$

Ecuación 6
Dónde, $E_{i}$ es el valor de energía total del intervalo calculado en la Ecuación 5, e $I_{i}$ es la intensidad de precipitación correspondiente. Al obtener el factor $R$, calculado de acuerdo a la metodología expresada anteriormente, los factores $K$, y $L S$, asociados con las propiedades físicas y topográficas, medidas en campo y las pérdidas de suelo $T$, medidas directamente en campo, se determinó el factor $C$ a partir de la Ecuación 1, obteniendo un valor medio para las pérdidas de suelo, asociadas a cultivos con corte por entresaque, parejo, y al caso de la condición sin cobertura vegetal o plantilla. Al despejar el factor $C$ de la Ecuación 1, se obtendría la expresión presentada en la Ecuación 7:

$$
C=\frac{T}{R K L S}
$$

Ecuación 7

Donde, $T$ es la pérdida correspondiente a las mediciones realizadas en campo y $R$ es el factor de erosividad de la lluvia, asociada a cada precipitación simulada, en $M J \mathrm{~cm} \mathrm{ha}^{-1} \mathrm{~h}^{-1}$, según el procedimiento descrito por las Ecuaciones 4, 5 y 6 , para estimar la erosividad de cada una de las intensidades de precipitación, simuladas con el equipo utilizado en campo. Por medio de un ANOVA y la determinación de diferencia entre medias de la prueba de Tukey, utilizando el software estadístico $R$ versión 3.4.1., se determinó la diferencia entre los sistemas de corte y la condición sin cobertura vegetal o plantilla.

Estimación de erosión potencial anual. Al calcular el factor $C$, para los dos sistemas de corte, obtenido en el paso anterior, se utiliza la Ecuación 1 , con un factor $R$ asociado a la erosividad potencial de las precipitaciones anuales, para determinar la pérdida potencial de suelo en un año. Se utilizó una expresión desarrollada por Rivera \& Gómez (1991), obtenida para el eje cafetero, que emplea el índice modificado de Fournier. Este tipo de expresiones permite estimar el factor $R$ en función de la precipitación total anual de una estación climática y, aunque no corresponde al departamento de Cundinamarca, es una de las pocas expresiones determinadas en Colombia. De esta forma, el factor $R$ correspondiente a la erosividad media anual para la región de estudio, se determinó por medio de la siguiente expresión:

$$
R=38,4(\overline{I M F})+28,3
$$

Ecuación 8

Donde, $R$ es el factor de erosividad expresado en $M J \mathrm{~mm} \mathrm{ha}^{-1}$ e es el índice modificado de Fournier que, en este caso, se tomó como el valor medio de una serie de precipitaciones de 30 años y se calculó por medio de la Ecuación 9:

$$
I M F=\sum_{1}^{12} \frac{\left(P_{i}\right)^{2}}{P}
$$

Ecuación 9

Donde, $P$ es la precipitación del mes $i$, y $P$ es la precipitación total del año. Al obtener el factor $R$ anual y los factores $C, K$ y $L S$ obtenidos previamente, se realiza una estimación de la 
erosión potencial para cada uno de los sistemas de corte y para el suelo sin cobertura vegetal o plantilla, reemplazando los valores obtenidos en la Ecuación 1.

\section{RESULTADOS Y DISCUSIÓN}

Los factores medios de erodabilidad presentados en la tabla 1 , para cada uno de los sistemas de corte y para el caso de la plantilla, en donde no se presenta una cobertura vegetal, se obtuvieron al reemplazar los códigos de tipo de permeabilidad y tipo de estructura seleccionados, así como los datos determinados para cada uno de los lotes de medición de contenidos de materia orgánica, arcilla, arena muy fina y limo; en la Ecuación 2, propiedades físicas, que también se presentan en la tabla 1. Aun cuando se seleccionaron lotes dentro de una misma finca, se debe tener en cuenta que la variabilidad dentro de los campos agrícolas es inherente a la naturaleza geológica de la formación de suelo y, en algunos casos, puede ser inducida por las prácticas de manejo (Monroy et al. 2017).

De acuerdo con la clasificación presentada por Moreno et al. (2017) y Proceedings of WEPP/WEPS (1995) los valores de erodabilidad presentados en la tabla 1, corresponden a grados de erodabilidad de bajos a moderados, con susceptibilidad escasa a moderada al escurrimiento, los cuales, al compararlos con otros estudios, presentan una mejor resistencia al desprendimiento y a la erosión, como es el caso de Moreno et al. (2017), quienes determinaron para suelos de textura franco-arcillosa a franco limosa, con estructura en bloques subangulares, valores de $K$ de 0,24 a 0,27, obteniendo mejores condiciones de erodabilidad que el caso del suelo sin cobertura, incluido en este estudio $(K=0,36)$. Contreras et al. (2015), en conglomerados y arenas aluviales, obtuvieron un $K$ de 0,48 , clasificando estos suelos como susceptibles al desprendimiento y la erosión. Los bajos niveles de limo en los suelos estudiados, así como los altos niveles de materia orgánica y permeabilidad, en especial, en el caso de los lotes cultivados bajo cualquier sistema de corte, permiten tener valores $K$, menores que los presentados en los estudios citados, lo que representa una mayor resistencia del suelo ante el desprendimiento y el escurrimiento superficial.

Aplicación de la Ecuación para determinar el factor C. Al seguir el procedimiento presentado en las Ecuaciones 4, 5 y 6 , con las intensidades de precipitación simuladas, se obtiene que, para $30,81 \mathrm{~mm} \mathrm{~h}^{-1}$, se obtuvo un valor $R$ expresado en $\mathrm{MJ} \mathrm{mm} \mathrm{ha}^{-1} \mathrm{~h}^{-1}$, correspondiente a 59,08 y de 131,79 y 438,37 , para intensidades de precipitación de $44,76 \mathrm{~mm}$ $\mathrm{h}^{-1}$ y $78,76 \mathrm{~mm} \mathrm{~h}^{-1}$, respectivamente, correspondientes a la energía cinética de las intensidades de precipitación aplicadas en el tiempo de duración de cada medición en campo. Generalmente, se presenta el factor $R$ en términos anuales, por lo cual, no se comparan estos resultados con otros valo- res de referencia de un intervalo más pequeño de aplicación de lluvia.

Al realizar el cálculo expresado en la Ecuación 9, para cada una de las mediciones realizadas, se obtuvieron valores medios de $C$ correspondientes a los dos sistemas de corte y a los momentos de medición, como se presentan en la tabla 2 , donde se observa una aproximación del comportamiento del corte por entresaque, con respecto al corte por parejo, observando que la erosión potencial es menor en este corte, al tener un factor $C$ dos veces menor, que el factor $C$ estimado, para el corte por parejo. Al realizar un análisis ANOVA entre los dos sistemas de medición, teniendo como factores de variación, tanto el sistema de corte como el momento en el que se realizó la medición, se obtuvo que solo el corte se resaltó como un factor real de variación con un estadístico $p$ de 0,001 , lo cual, representa una alta significancia al ser un valor menor del 0,05 , grado de significancia establecido para este tipo de análisis. Por su parte, no se resaltaron diferencias estadísticas significativas entre los momentos de medición, ni en la interacción de factores corte y momento. Estas diferencias, además se resaltaron con una prueba de Tukey, en la que, nuevamente, se estableció que la diferencia entre medias de los factores $C$, obtenidos bajo las mediciones de los dos sistemas de corte, representan una diferencia estadística significativa $(p=0,001)$.

Esta tendencia, también se observó en los momentos de medición, donde, en general, se observa un comportamiento en el que se obtienen factores $C$ mayores en corte por parejo que en corte por entresaque; sin embargo, no se percibe una clara variación del factor $C$ con respecto al grado de desarrollo del cultivo, pero sí se observa que la diferencia entre factores $C$ estimados para entresaque y parejo, se reduce a medida que se tiene un mayor grado de desarrollo del cultivo. Por su parte, el caso de la plantilla, al comportarse como un testigo de un suelo sin cobertura vegetal tiene una clara tendencia a presentar pérdidas de suelo superiores a cualquiera de los dos sistemas de corte, con un factor $C$ nueve veces mayor al valor medio obtenido, para el corte por parejo y casi 20 veces mayor al valor obtenido, para entresaque.

Al realizar un análisis ANOVA entre los resultados obtenidos bajo los dos sistemas de corte y el caso de suelo sin cobertura vegetal, se observó una diferencia entre cortes y tratamiento sin cobertura vegetal $\left(\mathrm{p}<2 \times 10^{-6}\right)$ y al comparar la diferencia entre medias, por medio de la prueba de Tukey, ya no se resaltó una diferencia entre cortes, ya que la mayor significancia se generó al comparar el caso de suelo sin cobertura vegetal, con cada uno de los cortes, obteniendo, en ambos casos, valores $\mathrm{p}$ por debajo de $1 \times 10^{-8}$. Al comparar estos valores con otros factores $C$, determinados para diferentes cultivos, se observa que el corte por parejo es similar al determinado para maíz con cobertura ( $C$ estimado de 
Tabla 2. Factores $C$, estimados para sistemas de corte y momentos de medición.

\begin{tabular}{|c|c|c|c|c|c|c|c|}
\hline \multirow{2}{*}{ Corte } & \multirow{2}{*}{ Momento } & \multicolumn{6}{|c|}{ Factor C } \\
\hline & & Media & $S_{d^{*}}$ & $\mathrm{CV}^{* * *}$ & Media & $S_{d^{*}}$ & $\mathrm{CV}^{* *}$ \\
\hline \multirow{3}{*}{ Entresaque } & Después del corte & 0,047 & 0,034 & 0,736 & \multirow{3}{*}{0,048} & \multirow{3}{*}{0,042} & \multirow{3}{*}{0,877} \\
\hline & A los 3 meses del corte & 0,054 & 0,050 & 0,928 & & & \\
\hline & A los 6 meses del corte & 0,044 & 0,043 & 0,974 & & & \\
\hline \multirow{3}{*}{ Parejo } & Después del corte & 0,114 & 0,152 & 1,332 & \multirow{3}{*}{0,105} & \multirow{3}{*}{0,126} & \multirow{3}{*}{1,200} \\
\hline & A los 3 meses del corte & 0,131 & 0,112 & 0,849 & & & \\
\hline & A los 6 meses del corte & 0,064 & 0,121 & 1,877 & & & \\
\hline \multicolumn{2}{|r|}{ Plantilla } & \multicolumn{4}{|c|}{0,978} & 0,815 & 0,833 \\
\hline
\end{tabular}

*S (Desviación estándar)

**CV (Coeficiente de variación)

0,1) o soja (C estimado de 0,17) (Gvozdenovich et al. 2017). Lianes et al. (2009) presentan valores de $C$, que van desde 0,003 , para bosque natural, 0,012 , para pastos de corte y 0,26 a 0,36 , para cultivos de papa con distintas rotaciones y los comparan con valores de $C$, obtenidos en informes técnicos presentados por la FAO, para cultivos en Costa Rica, donde se presenta para caña de azúcar un $C$ igual a 0,263.

Estimación de erosión potencial. Finalmente, se utiliza la Ecuación Universal de Pérdida de Suelo, presentada en la Ecuación 1, utilizando los factores $C$, estimados previamente, para las mediciones directas realizadas en corte por parejo, entresaque y en un lote en plantilla, sin cobertura vegetal. Además, por medio de las Ecuaciones 7 y 8 , se determinó el factor $R$, teniendo en cuenta los datos pluviométricos de una estación climatológica cercana al municipio de Quebradanegra, correspondiente a mediciones de precipitación mensual, en una serie de tiempo de 30 años (1980 - 2011). De esta forma, se obtuvo un $R$ de $6549,17 \mathrm{MJ} \mathrm{mm} \mathrm{ha}^{-1} \mathrm{~h}^{-1}$ que, de acuerdo con la clasificación presentada por Rivera \& Gómez (1991), corresponde a un valor de erosividad moderada.

Con este factor de erosividad, ya no asociado a un evento específico de precipitación, sino a la precipitación anual de la región de estudio, se obtuvo una aproximación a la erosión potencial por año, para cada uno de los cortes, obteniendo valores en $\mathrm{t} \mathrm{ha}^{-1} \mathrm{año}^{-1}$. Para el sistema de corte por entresaque, se obtuvo que la erosión potencial obtenida por aproximación de la USLE, para un suelo con contenidos de arcilla, de limo y de arena muy fina de $40,45 \%, 30,41 \%$, y $7,78 \%$, respectivamente, altos contenidos de materia orgánica, estructura en bloques, permeabilidad lenta a moderada e inclinación de $35 \%$, es de $12,35 \mathrm{tha}^{-1} \mathrm{año}^{-1}$. Para el caso del sistema de corte por parejo, en las mismas condiciones, se estimó una pérdida de $27,07 \mathrm{t} \mathrm{ha}{ }^{-1}$ año $^{-1}$. Finalmente, como una aproximación a un testigo, se estimó para plantilla, con- dición, en la cual, el suelo se encuentra descubierto, una pérdida estimada de $250,36 \mathrm{t} \mathrm{ha}^{-1}$ año $^{-1}$, observando que, en general, el cultivo de caña, para la producción de panela, ofrece una cobertura importante al suelo, en términos de protección contra eventos erosivos.

Al comparar estos resultados con la clasificación de pérdida de suelo citada por González et al. (2016), los valores estimados de erosión potencial en ambos sistemas de corte, se considerarían moderados, al encontrarse en el rango de 10 a $50 \mathrm{t} \mathrm{ha}{ }^{-1} a n ̃ o^{-1}$. Por su parte, las pérdidas en el caso del suelo sin cobertura vegetal, se consideraría una pérdida muy alta, al estar por encima de 200t ha ${ }^{-1}$ año ${ }^{-1}$. Pruebas realizadas en kikuyo, cobertura vegetal y suelo descubierto, adelantadas por Álvarez \& Fernández (2009), determinaron que el suelo descubierto permitía un arrastre por sedimentos $57 \%$, mayor que en el caso de la cobertura vegetal y $80 \%$, mayor que en el caso de kikuyo, ya que el cultivo contribuye a dar fuerza, estabilidad estructural y favorece la infiltración del agua.

De acuerdo con la estimación del factor $C$ y de la erosión potencial anual para los dos sistemas de corte de caña panelera, se determinó que las pérdidas de suelo, bajo corte por parejo, son mayores que las que se presentaron en corte por entresaque; además, esta diferencia entre sistemas de corte, se obtuvo por medio de análisis de varianza y por la prueba estadística de Tukey de diferencia de medias. Por otra parte, se observó que las diferencias entre factores de manejo $C$, estimados en ambos tipos de corte, disminuyeron a medida que el cultivo tuvo un mayor grado de desarrollo, por lo que la mayor diferencia entre factores $C$, en ambos sistemas de manejo, se presentó en el momento justo después del corte; sin embargo, la diferencia entre momentos de medición y entre la interacción corte-momento, no se resaltó por medio de análisis estadístico. 
Al comparar los resultados de erosión potencial con un suelo sin cobertura vegetal, se muestra el potencial del cultivo de caña panelera, bajo ambos sistemas de manejo, para proteger el suelo ante eventos erosivos, al observar que, aún en el caso del corte por parejo, la remoción de suelo por escorrentía superficial es aproximadamente nueve veces menor que la que se tendría en un suelo sin cobertura vegetal. Esta reducción, como explican Álvarez \& Fernández (2009), se debe a que la cubierta vegetal del suelo ayuda a la retención del agua y atenúa el impacto de las gotas de lluvia. Esta protección del suelo, se presenta para las características de manejo que se dan en Cundinamarca, en donde, tanto el cultivo por parejo como por entresaque, mantiene constantemente la cobertura de hojarasca, producto de desechos de cosechas anteriores o de la pérdida de hojas por la planta, en el ciclo productivo.

Agradecimientos. A la Corporación Colombiana de Investigación Agropecuaria (AGROSAVIA) y a todo el equipo de trabajo del producto "Indicadores de impacto ambiental en la agroindustria panelera". Conflictos de intereses: El manuscrito fue preparado y revisado con la participación de todos los autores, quienes declaramos que no existe conflicto de intereses que ponga en riesgo la validez de los resultados presentados. Financiación: Este estudio fue financiado por la Corporación Colombiana de Investigación Agropecuaria (AGROSAVIA).

\section{REFERENCIAS}

1. ÁLVAREZ-HERRERA, J.F.; FERNÁNDEZ, J.P. 2009. Evaluación de la erosión de un inceptisol de Tunja con diferentes coberturas al impacto de lluvias simuladas. Ingeniería e investigación. 29(3):86-91.

2. ARNOULDS, H. 1977. Predicting soil losses due to sheet and rill erosion. En: FAO Conservation Guide No. 1: Guidelines for watershed management. p.99-123.

3. CONTRERAS, E.; PORTUGUEZ, M.; MUÑOZ, A.; CONDORHUAMAN, M. 2015. Inseguridad de la producción agrícola de alimentos por erosión hídrica en la cuenta del Río Siguas - Región Arequipa. Ciencia e investigación. (Perú). 18(1):26-32.

4. DERCON, G.; MABIT, L.; HANCOCK, G.; NGUYEN, M.L.; DORNHOFER, P.; BACCHI, O.O.S.; ZHANG, X. 2012. Fallout radionuclide-based techniques for assessing the impact of soil conservation measures on erosion control and soil quality: an overview of the main lessons learnt under an FAO/IAEA Coordinated Research Project. J. Environmental Radioactivity. 107:78-85. https://doi.org/10.1016/j.jenvrad.2012.01.008
5. GARCÍA, H.; ALBARRACÍN, L.; TOSCANO, A.; SANTANA, N.; INSUASTY, O. 2007. Guía Tecnológica para el Manejo Integral del Sistema Productivo de Caña Panelera. Corporación Colombiana de Investigación Agropecuaria, AGROSAVIA. Ministerio de Agricultura y Desarrollo Rural. Produmedios. Bogotá, D.C. 152p.

6. GARCÍA, F.; TERRA, J.; SAWCHIK, J.; PÉREZ, M. 2017. Mejora de las estimaciones con USLE/RUSLE empleando resultados de parcelas de escurrimiento para considerar el efecto del agua del suelo. Rev. Agrociencia. (Uruguay). 21(2):100-104.

7. GOBIN, A.; JONES, R.; KIRKBY, M.; CAMPLING, P.; GOVERS, G.; KOSMAS, C.; GENTILE, A.R. 2004. Indicators for pan-European assessment and monitoring of soil erosion by water. Environmental Science \& Policy. 7(1):25-38. https://doi.org/10.1016/j.envsci.2003.09.004

8. GONZÁLEZ, O.; BOJÓRQUEZ, J.; FLORES, F.; MURRAY, R.; GONZÁLEZ, A. 2016. Riesgo de erosión hídrica y estimación de pérdida de suelo en paisajes geomorfológicos volcánicos en México. Rev. Cultivos tropicales. (Cuba). 37(2):45-55.

9. GVOZDENOVICH, J.J.; PÉREZ, M.; NOVELLI, L.E.; BARBAGELATA, P.A. 2017. ¿Puede WEPP mejorar la predicción de la erosion de suelos respecto a USLE? Rev. Ciencia del Suelo. (Argentina). 35(2):259-272.

10. LIANES, E.; MARCHAMALO, M.; ROLDÁN, M. 2009. Evaluación del factor $C$ de la RUSLE para el manejo de coberturas vegetales en el control de la erosion en la Cuenca del Río Birrís, Costa Rica. Rev. Agronomía Costarricense 33(2):217-235.

11. MONROY-RODRÍGUEZ, F.L.; ÁLVAREZ-HERRERA, J.G.; ALVARADO-SANABRIA, O.H. 2017. Distribución espacial de algunas propiedades físicas del suelo en un transecto de la granja Tunguavita, Paipa. Rev. U.D.C.A. Act. \& Div. Cient. 20(1):91-100. https://doi. org/10.31910/rudca.v20.n1.2017.66

12. MORENO, R.; CAMPOS, P.; AVENDAÑO, J.; NÚÑ̃EZ, V.; GIL, N.M.; SALAS, A.; JOEL, E. 2017. Distribución espacial y análisis de la pérdida de suelo en microcuencas de la Sierra de Vaqueros (Salta, Argentina) mediante el uso de un SIG. Rev. Espacio y Desarrollo. (Argentina). 30(2017):161-192. https://doi. org/10.18800/espacioydesarrollo.201702.007 
13. PANAGOS, P.; BORRELLI, P.; MEUSBURGER, K. 2015. A new European slope length and steepness factor (LS-Factor) for modeling soil erosion by water. Geosciences, 5:117-126. https://doi.org/10.3390/geosciences5020117

14. Proceedings of WEPP/WEPS. 1995. Symposium, August 9-11, Des Moines, IA, Soil and Water Conservation Society, Ankeny, IA.

15. RIVERA, J.; GÓMEZ, A. 1991. Erosividad de las lluvias en la zona cafetera central colombiana (Caldas, Quindio y Risaralda). Cenicafe. 42(2):37-52.

16. SHAKESBY, R.A. 2011. Post-wildfire soil erosion in the Mediterranean: Review and future research directions. Earth-Science Reviews, 105(3-4):71-100. https://doi.org/10.1016/j.earscirev.2011.01.001
17. WILLIAMS J. 1975. Sediment routing for agricultural watersheeds. Water Resour. Bull. 11(5):965-974.

18. WISCHMEIER, W.H.; SMITH, D.D. 1978. Predicting erosion losses, a guide to conservation planning. Washington: USDA. (Agricultural Handbook 537). Washington D.C. 67p.

19. WISCHMEIER, W.H.; SMITH, D.D. 1960. An Universal Soil Loss Estimating Equation to Guide Conservation Farm Planning. 7th International Congress of Soil Sciences. Soil Science Society. vol. 1, p.418425.

Recibido: Abril 19 de 2018

Aceptado: Octubre 22 de 2018

Cómo citar:

Tauta Muñoz, J.L.; Camacho-Tamayo, J.H.; Rodríguez Borray, G.A. 2018. Estimación de erosión potencial bajo dos sistemas de corte de caña panelera utilizando la ecuación universal de pérdida de suelos. Rev. U.D.C.A Act. \& Div. Cient.21(2):405-413. https://doi.org/10.31910/rudca.v21.n2.2018.1074 